\title{
Diseño e implementación de un sistema hospitalario basado en tecnología RFID: "Computación ágil, inteligente y móvil
}

\section{Desing and implementation of a hospital system based on technology RFID "Agile, intelligent and mobile Computation"}

Fecha de recepción: 28 de marzo de 2008 Fecha de aceptación: 14 de julio de 2008
Carlos Alberto Escobar De Lima', Iván Rodrigo Vargas Ramírez², Alejandro Quintero Acevedo ${ }^{3}$, Alberto Acosta López ${ }^{4}$

\section{Resumen}

Los sistemas de identificación por radio frecuencia (RFID) hacen parte de las tecnologías emergentes que nuestro país necesita implementar en el sector de la salud para optimizar los procesos que conforman los principales cuellos de botella, como el manejo, identificación, registro, gestión y control de las historias clínicas de cada paciente. Por ende, es indispensable crear soluciones robustas basadas en nuevos paradigmas vanguardistas fabricados desde la investigación académica.

Gracias al grupo de investigación TRHISCUD (Tratado de Historias Clínicas de la Universidad Distrital) hemos diseñado y desarrollado una solución para los sistemas hospitalarios a través de la automatización de las historias clínicas por medio de módulos informáticos que mejoren la agilidad, autenticidad y precisión en el acceso a la información a través de tarjetas inteligentes que permitan la movilidad eficiente de los datos para una gestión efectiva por parte de la administración del sistema.

Gracias a esto, el personal del hospital puede disponer de información crítica en tiempo real, en momentos donde un error puede convertirse en artífice de una tragedia.

\section{Palabras clave}

Historias clínicas, información crítica, RFID, sistemas de identificación por radio frecuencia, tecnología emergente.

1 Ingeniero de Sistemas, Universidad Distrital F.J.C. Estudiante Maestria en Ciencias de la Informacion y las comunicaciones, Universidad Distrital. Monitor del grupo de investigación TRHISCUD, Tratado de Historias Clínicas, proyecto de Telemedicina.

2 Ingeniero de Sistemas Universidad Distrital F.J.C. Ingeniero Industrial Pontificia Universidad Javeriana. Estudiante de Maestría Logistica Industrial. Universidad Politécnico de Torino. Monitor del grupo de investigación TRISCUD, Tratado de Historias Clínicas, proyecto de Telemedicina.

3 Ingeniero de Sistemas (Estudiante de X semestre), Universidad Distrital F.J.C. Monitor del grupo de investigación TRHISCUD, Tratado de Historias Clínicas, proyecto de Telemedicina.

4 Ingeniero de Sistemas, Universidad Distrital, Licenciado en Matematicas, MsC Teleinformática, Director del grupo de investigación TRHISCUD ("Tratamiento de Historias Clínicas de la Universidad Distrital") aprobado y reconocido en COLCIENCIAS desde 1991. 


\section{Abstract}

The systems of identification by radio frequency (RFID) are part of the emergent technologies that our country needs to implement in the sector of the health to optimize the processes that conform the main necks of bottle, as they are it the handling, identification, registry, management and control of clinical histories of each patient. Therefore he is indispensable to create robust solutions based on new made vanguar-distas paradigms from the academic investigation.
By means of the group of investigation TRHISCUD ("Treaty De Clinical Histories Of the Distrital University") we have designed and developed a solution for the hospitable systems to traverse of the automatization of clinical histories by means of computer science modules that improve the agility, authenticity and precision in the access to the information through smart cards that they allow to the efficient mobility of the data for an effective management on the part of the system management.

Thanks to this, the personnel of the hospital can have critical information in real time, at moments where an error can become creator of a tragedy.

TABLA I. POSICIONAMIENTO DE RFID

\begin{tabular}{|c|c|c|}
\hline thr & 896u & \\
\hline 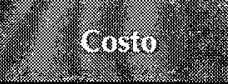 & $\begin{array}{l}\text { Miles de } \\
\text { dólares. }\end{array}$ & $\begin{array}{l}\text { Cada tag tiene un valor de } 0.20 \\
\text { dólares. }\end{array}$ \\
\hline 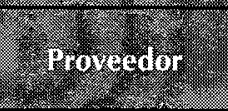 & $\begin{array}{l}\text { Fuerzas } \\
\text { Armadas } \\
\text { Británicas. }\end{array}$ & $\begin{array}{l}\text { Brasil, México, Estados Unidos, } \\
\text { España, Israel y Alemania. }\end{array}$ \\
\hline 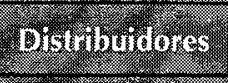 & $\begin{array}{l}\text { Fuerzas } \\
\text { Armadas. }\end{array}$ & $\begin{array}{l}\text { En Colombia existen más de } 12 \\
\text { empresas. }\end{array}$ \\
\hline Aviligentin. & Guerra. & Comercio en general. \\
\hline $\begin{array}{l}\text { Bisporigunal } \\
\text { Comercio }\end{array}$ & Privado. & \\
\hline
\end{tabular}

\section{TABLA 2. APLICACIÓN DE RFID EN COLOMBIA}

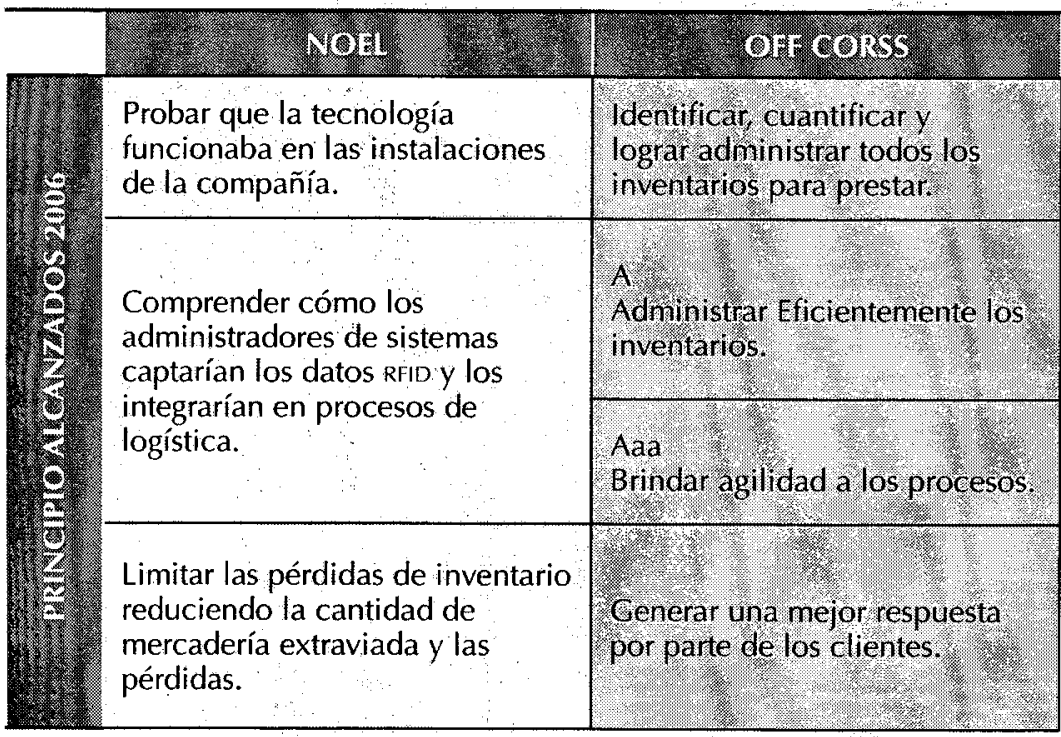

\section{Key words}

Clinical histories, critical information, RFID, systems of identification by radio frequency, emergent technology.

\section{Introducción}

Los sistemas RFID (Radio Frequency Identification) son una de las tecnologías que hoy en día se están probando por medio de prototipos para nuevas $\mathrm{y}$ prometedoras aplicaciones en muchos campos de investigación y desarrollo.

Nació por la necesidad de los británicos de identificar aviones en la segunda guerra mundial, existen referencias de 1960 donde se afirma su aparición en la industria armamentista americana, pero por sus elevados costos no se había popularizado en otras áreas del comercio [1]. 
Ahora encontramos que el nivel de producción y adquisición se está incrementando al punto de que en Colombia empieza a posicionarse en el mercado.

Hoy en día los RFID están siendo implementados en múltiples organizaciones, simulando actividades a través de nuevos dispositivos inalámbricos con gran potencial de comunicación como lo fue desarrollado en dos de las compañías más exitosas de Colombia. [2]

La aceptación de los RFID como tecnología innovadora de actividades y procesos, está evolucionando positivamente, ya que cuenta con sistemas sofisticados que funcionan, desde identificación

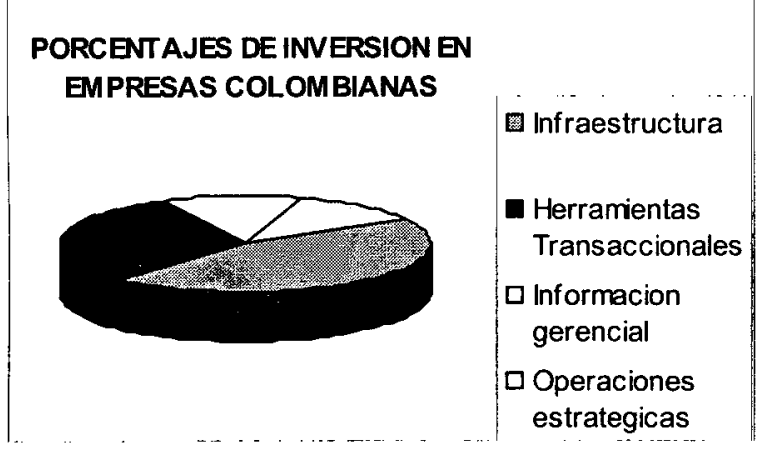

de animales (ganado vacuno), personas y equipos hasta la seguridad y control de acceso en organizaciones. "La tecnología RFID tiene el potencial de brindarnos una ventaja competitiva real, ayudar a mejorar el cumplimiento de los pedidos e incrementar la satisfacción del cliente" [3].

Desde la investigación universitaria hemos analizado y diseñado una propuesta metodológica para el desarrollo de este proyecto tecnológico, por ende buscamos masificar la información adquirida para fomentar el uso de RFID y su infinidad de aplicaciones a nivel local e internacional.

Con la cultura de globalización y libre comercio en los productos y servicios, es importante destacar la necesidad de que nuestras PYMEs (pequeñas y medianas empresas) conozcan este tipo de información para conectar sinérgicamente la tecnología con el talento humano a través de la identificación por radiofrecuencia, para mejorar su calidad y así mismo nivel de competitividad. En plena revolución del conocimiento y de la tecnología, es indispensable fomentar el proceso de actualización o adquisición de nuevos sistemas para la prestación de servicios por parte de las organizaciones a través de dispositivos que permitan el mejoramiento continuo en la organización, atacando los problemas que generan la incertidumbre y el riesgo informático. Como se ve reflejado en la siguiente distribución del presupuesto promedio de las empresas nacionales. [4].

El modelo colombiano fue diseñado para la clasificación de los hospitales de acuerdo a unos parámetros, como los siguientes [5]:

Nivel I: consulta externa y hospitalización en medicina general. Compuesto por cerca de tres mil 
puestos, centros de salud y hospitales locales.

Nivel II: consulta externa, cirugía general, ginecología y obstetricia, medicina interna, pediatría y especialidades quirúrgicas con requerimiento intermedio de hospitalización. Aproximadamente cien hospitales regionales están en esta categoría.

Nivel III: consulta externa, cirugía general, ginecología y obstetricia, medicina interna, pediatría y todas las especialidades y subespecies, cualidades del nivel avanzado de hospitalización. Doce hospitales universitarios y cuarenta instituciones especializadas integran esta categoría.

La gestión y control de las historias clínicas y de los servicios médicos generales en el mundo entero es un problema colateral, por ejemplo se estima que por este concepto en Estados Unidos se producen más de un millón de lesiones y cien mil muertes anuales, las cuales se hubiesen podido evitar al mejorar la calidad en la atención de los pacientes. A pesar de estas alarmantes cifras, se estima que la mayoría de los errores médicos no resulta en lesiones graves o muerte, sino que tienen consecuencias que pasan inadvertidas-dado el estado clínico del paciente, pero sumamente costosas para los sistemas de salud. [6]

Es una realidad que las empresas colombianas deban buscar mejorar sus servicios sin descuidar la

Fuente: [9]

Fuente: [10]
TABLA 4. Ventajas y desventajas de RFID:

\begin{tabular}{|c|c|}
\hline $\begin{array}{l}\text { Automatiza, eliminando el error } \\
\text { humano en la identificación, conteo, } \\
\text { rastreo, clasificacion y ruteo de } \\
\text { cualquier objeto. }\end{array}$ & $\begin{array}{l}\text { A diferencia del código de barras, } \\
\text { RFID puede ser rastreado a distancia } \\
\text { después de salir del supermercado. }\end{array}$ \\
\hline $\begin{array}{l}\text { Elimina errores por } \\
\text { irresponsabilidad. }\end{array}$ & \multirow{3}{*}{$\begin{array}{l}\text { El alto costo de los equipos lectores } \\
\text { y del software, la poca precisión } \\
\text { en el ingreso de datos (mientras el } \\
\text { código de barras supera el } 99,9 \% \\
\text { de confiabilidad, algunas etiquetas } \\
\text { de radiofrecuencia caen incluso por } \\
\text { debajo del } 90 \% \text { ). }\end{array}$} \\
\hline $\begin{array}{l}\text { Elinina errores y por consec } \\
\text { el desperdicio de recursos. }\end{array}$ & \\
\hline herementa el control de calidad ? & \\
\hline $\begin{array}{l}\text { Increasentan la eficiencia y la } \\
\text { productivicad de la empresa. }\end{array}$ & $\begin{array}{l}\text { Dificultades de los lectores de radio } \\
\text { para detectar algunos productos } \\
\text { dentro de las cajas, e interrupciones } \\
\text { de la señal cuando es reflejada por el } \\
\text { metal o absorbida por el agua. }\end{array}$ \\
\hline
\end{tabular}

\section{TABLA 5. COMPONENTES RFID}

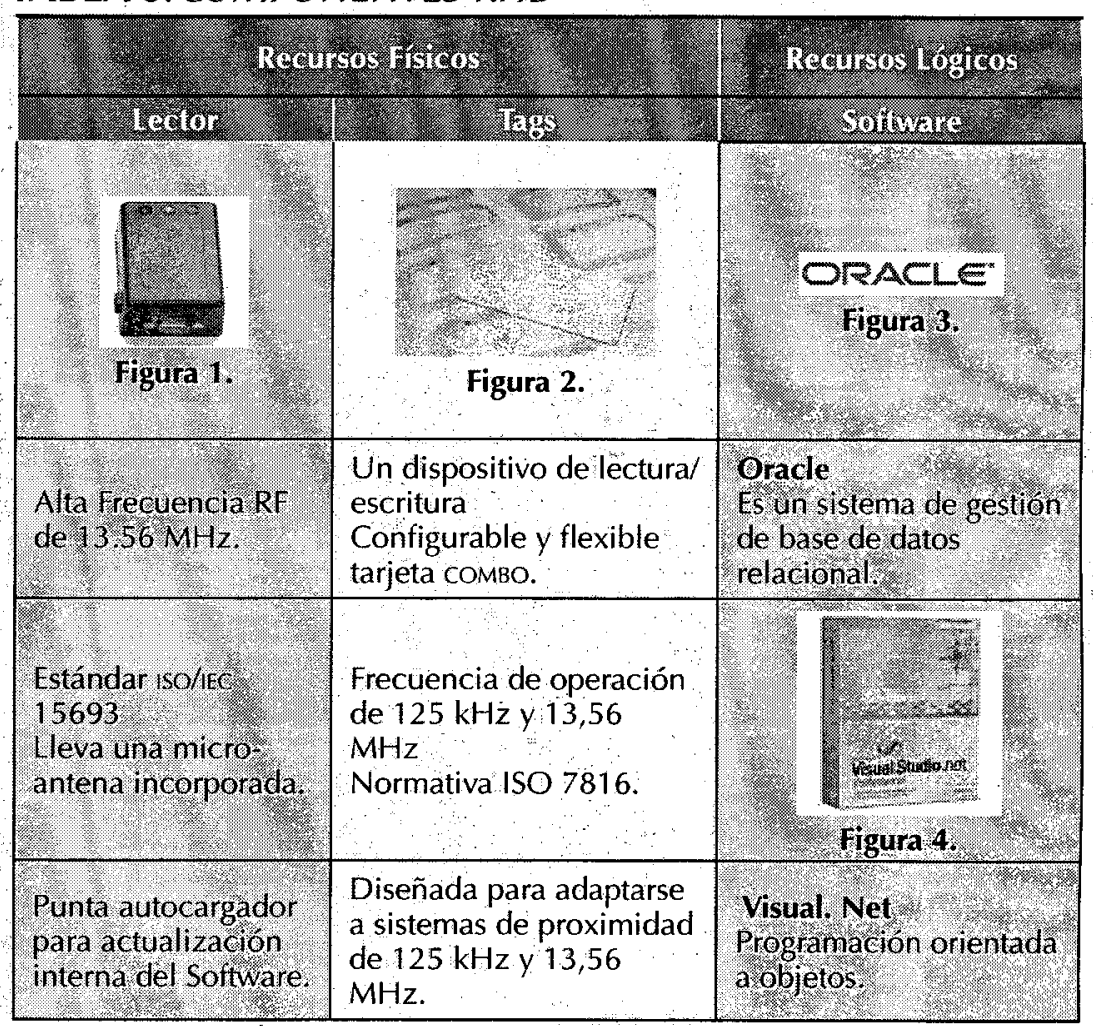

calidad de sus recursos y productos; el prototipo se desarrolló con el fin de automatizar las operaciones internas de un sistema hospitalario por medio. 
de la optimización, control y gestión de procesos, con la ayuda de la tecnología RFID.

\section{Características del sistema de identificación por radio frecuencia}

La tecnología RFID permite asignar a cualquier producto una identificación por medio de tags (chips únicos de identificación), productos que se están implementados como estándares empresariales. En la actualidad el código de barras, por su precio y manejo, está posicionado en el mercado de una manera fuerte; sin embargo, se están atacando sus fortalezas a través de cambios en los paradigmas; ejemplo claro de ello es la revolución de los sistemas de RFID que poseen características únicas, como la capacidad de ser programable y su amplitud en los niveles de seguridad, además de que logra ser capaz de suplir muchas más aplicaciones actuales y barreras que otros sistemas de identificación enfrentan ante la complejidad de las organizaciones empresariales [7].

Cuando un código de barras no es leído desde la primera vez genera tiempos extra de espera, colas en los puntos de pago, aumentos de costos de operación y reduce los beneficios y la verdadera eficiencia. En cambio, un sistema basado en radiofrecuencia tiene una cobertura mayor y un porcentaje de error mucho menor [8].

\section{Cómo funciona el RFID:}

Un sistema de identificación por radiofrecuencia está formado por tres elementos básicos.

Un tag es un micro-chip incorporado con una antena, que puede adherirse a casi cualquier medio (madera, plástico, e incluso metales). El microchip almacena datos dependiendo de las capacidades del fabricante; cada tag tiene un único número que lo identifica, como los códigos electrónicos de cada producto (EPC) que son desarrollados por estándares internacionales de EPC GLOBAL o como las direcciones MAC de las tarjetas de red computacionales, lo que proporciona una única identificación en el mundo [11].

El sistema funciona de la siguiente manera: el lector envía una serie de ondas de radiofrecuencia que son captadas cuando el tag entra en el radio de frecuencia enviado por el lector, y son obtenidas por la micro antena de éste. Dichas ondas activan el microchip, el cual, a través de la micro antena y mediante ondas de radiofrecuencia, transmite al lector los datos solicitados por el lector, que pueden ser desde el cu (código único), hasta datos específicos almacenados, como nombres de equipos, pacientes, muestras entre otros. Finalmente, el lector recibe el dato o los datos, si son varios tag identificados al mismo tiempo, y los envía al PC, donde son diseccionados hacia una aplicación desarrollada en .Net, para la autenticación en la aplicación dependiendo del tipo de dato o código que contenga el tag (pacientes, muestras, equipos, etc.), de manera que se pueda acceder a la base de datos, ver los datos previamente registrados, y así procesar la información de forma efectiva ante las necesidades del entorno específico [12].

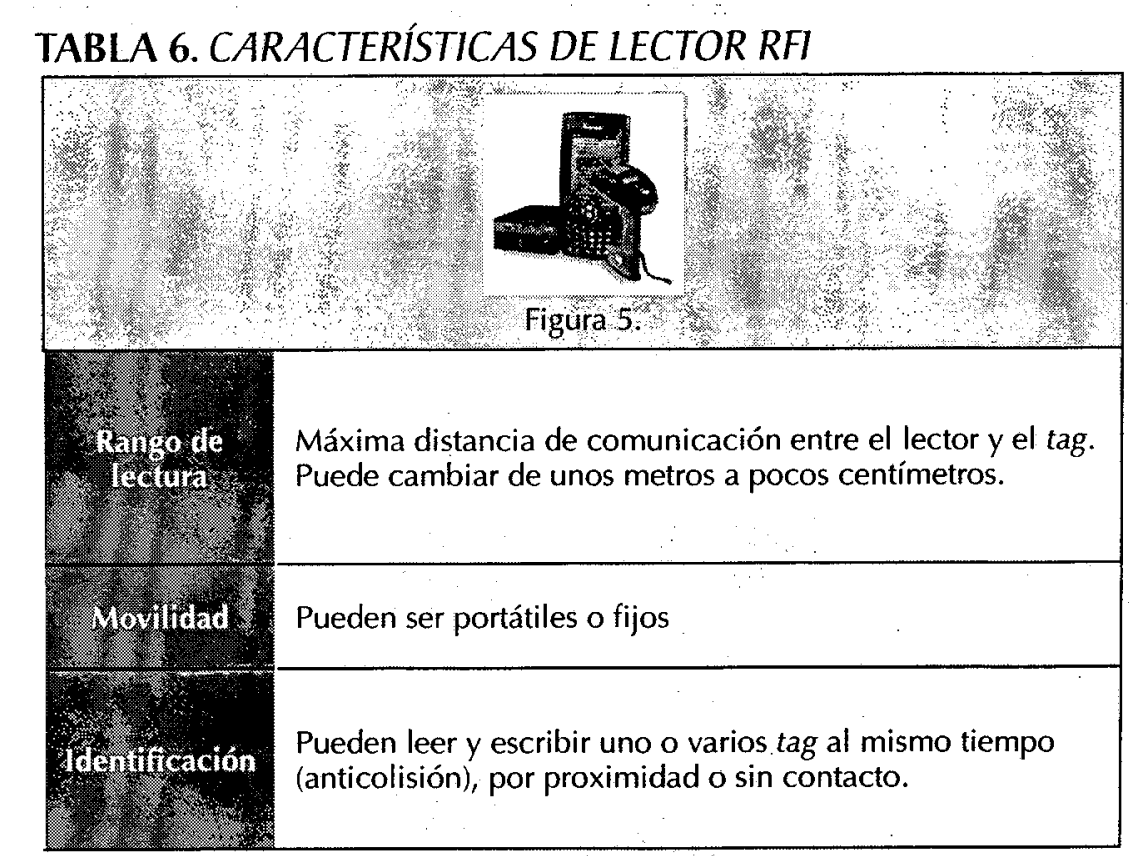




\section{Lectores RFID}

Los lectores son dispositivos encargados de transmitir y receptar los datos por medio de radio frecuencia mediante una antena; los lectores poseen un modulo de acoplamiento entre el tag y el lector, una unidad de control y un terminal para la comunicación con el computador.

\section{Transponders o tags pasivos y activos}

Los trasponders o tag son unos dispositivos compuestos por un microchip y una antena, utilizados para la

TABLA 7. Diferencias entre las clases de los Transponders

\begin{tabular}{|c|c|c|c|}
\hline $\begin{array}{l}\text { Tag de } \\
\text { identificacion } \\
\text { pasivo (usable } \\
\text { en un rango de } 3 \\
\text { metros) }\end{array}$ & $\begin{array}{l}\text { Identificación } \\
\text { pasiva y tag con } \\
\text { memoria (usable } \\
\text { en un rango de } 3 \\
\text { metros). }\end{array}$ & $\begin{array}{l}\text { Tag asistidas por } \\
\text { bateria pasivas. } \\
\text { a }\end{array}$ & $\begin{array}{l}\text { Tag de batería } \\
\text { activas (tags } \\
\text { trasmitir } \\
\text { portadora). }\end{array}$ \\
\hline $\begin{array}{l}\text { Backscalter } \\
\text { (habla de } \\
\text { primero el } \\
\text { interrogador) }\end{array}$ & $\begin{array}{l}\text { Backscatter } \\
\text { (habla de } \\
\text { primero el } \\
\text { interrogador). }\end{array}$ & $\begin{array}{l}\text { Más } \\
\text { funcionalidad } \\
\text { en el chip } \\
\text { nemoria, } \\
\text { sensores, etc }\end{array}$ & $\begin{array}{l}\text { Transmisión } \\
\text { activa } \\
\text { (Permite Tag- } \\
\text { hablar- primero } \\
\text { modos de } \\
\text { operación). }\end{array}$ \\
\hline Muy bajo costo & Seguridad. & $\begin{array}{l}\text { Backscatter } \\
\text { Gabla de } \\
\text { primero el } \\
\text { nterrogador. }\end{array}$ & $\begin{array}{l}1.00 \text { metros de } \\
\text { rango. }\end{array}$ \\
\hline 3 & Muy bajo costo: & Costo moderado & Alto costo. \\
\hline
\end{tabular}

Fuente [13]

transmisión de datos en RFID. Se pueden encontrar en varias formas y tamaños como (etiquetas, manillas, tarjetas) las cuales son implantadas en el objeto o persona que se va a identificar.

Cuando se inicia comunicación con el lector, el tag, por medio de la antena, envía una serie de datos del objeto identificado, como temperatura, parámetros, número de identificación, los cuales entran a una base de datos específica que depende de la aplicación.

\section{Software}

Una característica importante es que cada uno de los tag tiene un único número de serie, de fábrica, que es inalterable y distinto a los demás, comúnmente llamados como lectura de número único (Read only number). El rango de banda de frecuencia en el que trabaja varía entre de $60 \mathrm{kHz}$ a $5.8 \mathrm{GHz}$; existen dos tipos de bandas, la de baja frecuencia (LF) que está entre 125 y $134 \mathrm{KHz}$ y la de alta frecuencia $\mathrm{HF}$ 13.56 MHz. En la RFiD los tag han sido clasificados por la EPC (Electronic Code Product), su forma de funcionamiento se divide en cuatro clases:

Clase 0, 1 y 2: Estos tipos de tag pasivas no poseen una batería propia, entonces lo que realizan es la obtención de la energía de RF que envía al lector y la extraen.

Clase 3: Este tipo de tag asistido por batería pasiva, son tag que ofrecen un mayor rango y grado de fiabilidad que la clase 0,1 y 2 de los tag pasivos.

Clase 4: A diferencia de los tags pasivos, los activos tienen la propiedad de que cualquiera de los dos componentes pueden iniciar la comunicación ya sea el lector o el tag. Las etiquetas activas tienen una radio onboard, lo que es indicador de un rango mucho mayor que el de las pasivas [13].

El sistema de RFID es flexible al software de programación que se implemente ya que únicamente se comunica a través de variables definidas por tramas de información; esta ventaja absoluta de esta tecnología crea un núcleo infinito de futuras aplicaciones a desarrollar, en donde sea viable la utilización de la identificación de elementos. 


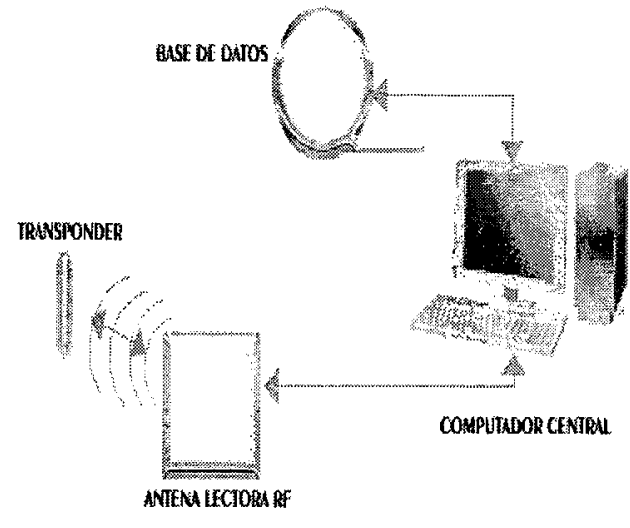

Figura 6. Sistema del prototipo.

El tag envía datos al lector, el lector recibe esos datos y los direcciona hacia el computador donde pueden ser manipulados y almacenados en aplicaciones que existen actualmente y/o que se desarrollan para una aplicación especifica.

Por ejemplo, los sistemas de bases de datos en Oracle , MySQL y lenguajes de programación como Java, Visual.net, son algunos de los sistemas de desarrollo en los cuales el RFID interactúa sin ningún problema, seleccionándolos según los requerimientos y características de la organización.

\section{Aplicación al prototipo}

El prototipo se realizó utilizando un tag para la identificación de cada paciente con un único código capaz de identificarlo y reconocerlo en el lector, para ser enviado a la aplicación y poder ser autenticado en la base de datos.

La aplicación Serial Port, que maneja el protocolo rs232 para la comunicación del lector RFID, está desarrollada en C\# y la aplicación encargada de la gestión de la base de datos está en ASP.NET, gracias a que estos lenguajes son de Visual Studio.NET, estudiados distintas formas de enviar el dato que captura el Serial Port desde C\# y recibirlo en ASP. net, en los campos donde es necesario capturar el lo que es enviado por el lector.

\section{Modelo del prototipo}

El-modelo del sistema utilizado para el desarrollo del prototipo fue diseñado en base a requisitos tomados previamente en la fase de análisis de requerimientos del modelo espiral, como se ve en la figura siguiente:

\section{Metodología del proyecto}

Debido al uso de una nueva tecnología, los riesgos técnicos en el desarrollo del sistema fueron altos por naturaleza. Un error en su funcionamiento interno podría significar la pérdida de vidas humanas en un centro hospitalario. Es por ello que un error en el producto constituye un alto riesgo para la misión del mismo, además del alto nivel de entropía que maneja la organización debido al volumen del sistema.

La metodología del proyecto consiste en realizar incrementos progresivos a medida de que se desarrolla el sistema y probar los resultados con cada nuevo incremento, para de este modo poder identificar los errores en la implementación, obtener los datos para depurarlos, reconocer y hacer un análisis de los riesgos potenciales que pueden surgir, de manera que se puedan tomar las decisiones apropiadas con el fin de mejorar la sinergia entre los diferentes elementos.

\section{Ventajas del modelo en espiral para el desarrollo del sistema hospitalario basado en RFID}

El modelo en espiral proporciona el potencial para el desarrollo rápido de versiones incrementales del software [14], donde los riesgos son un factor crítico.

Debido a su naturaleza iterativa, a medida que avanza el proyecto, tanto cliente como desarrollador logran una mayor compresión de los riesgos en cada una de las iteraciones, de manera que son capaces de reaccionar más efectivamente ante ellos con cada nuevo ciclo en la espiral. Además, si se aplica adecuadamente, el modelo debe reducir los riesgos antes de que se conviertan en verdaderos problemas, 
ya que demanda una consideración directa de los riesgos técnicos en todas las etapas del proyecto [14].

Otro factor de gran importancia es el hecho que el modelo en espiral permite el uso de prototipos en las distintas etapas del desarrollo del software. Debido a que este va a ser utilizado por una gran variedad de usuarios, desde operarios, hasta enfermeras y doctores, es importante que se tenga la posibilidad de disponer de prototipos, con el fin de que tales usuarios puedan evaluar las funcionalidades del sistema, su facilidad de uso y su correcto funcionamiento; de esta manera, pueden irse refinando los requisitos del software, así como identificando los errores en el mismo, de manera que ayude a reducir los riesgos técnicos del producto, al ir perfilando y definiendo dichos requisitos con las retroalimentaciones obtenidas con cada prototipo.

\section{Sistema de salud colombiano}

El sistema hospitalario colombiano en la actualidad cuenta con proyectos en los hospitales públicos de digitalización de historias clínicas de cada paciente y módulos que buscan alcanzar y registrar estándares de calidad entre los diferentes procesos del sistema, pero la identificación sigue realizándose por medio de una carnetización que tiene niveles bajos de seguridad e interactividad con el sistema hospitalario. En cambio, el sector privado se está enfocando básicamente a la adquisición de mejores equipos electrónicos para la seguridad y mejor prestación de servicios para la comunidad en general. Por esto, tanto el sector privado y público necesitan implementar un sistema de información capaz de identificar, diferenciar y gestionar a los pacientes según un registro único que le permita tener una especie de tranzabilidad en el centro médico a través de historias clínicas electrónicas.

Los sistemas de identificación por radio frecuencia tienen la oportunidad y están llamados para trabajar directamente todos y cada uno de los cuellos de botella a través de un mejor control de inventarios, de una autenticación sistemática de los pacientes por medio de carné con chips inteligentes que conserven la información necesaria para cualquier causa de accidentalidad y seguridad para el diagnóstico y análisis prequirúrgico.

\section{Gestión actual de las historias clínicas}

En la actualidad, gran parte de los centros hospitalarios en Colombia aún manejan el registro de las historias clínicas de la manera tradicional, es decir, aún llevan estos registros en papel. Sin embargo, se están dando iniciativas para implementar tecnologías que permitan crear sistemas de información completos, con el fin de tener la capacidad de manejar de una manera más óptima, toda la información que se genera en una entidad hospitalaria, y así mejorar la calidad de la atención a los pacientes.
TABLA 8. Ventajas del modelo en espiral para RFD en historias clínicas electrónicas

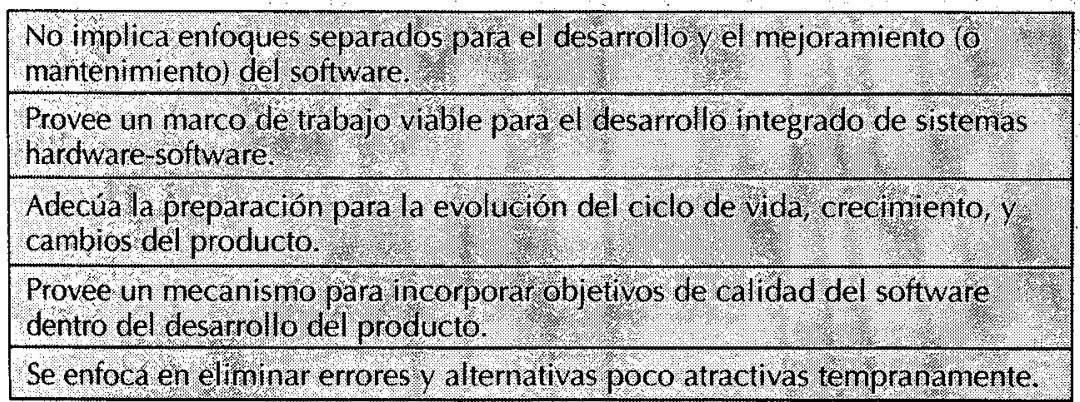

Fuente [15]
Una estrategia innovadora que utiliza recursos tecnológicos para producir un servicio diferenciador que generará una ventaja competitiva en el medio de las clínicas privadas en Colombia es el de la digitalización de la Clínica Fundación Santa Fe de Bogotá con el objetivo de mejorar la calidad de la prestación del servicio de salud a la población. 


\section{Necesidades en los hospitales}

Los principales requerimientos que los hospitales necesitan hoy en día tanto en Bogotá como el resto del país se representan a continuación, teniendo en cuenta el uso de la tecnología de sistemas de identificación por radio frecuencia RFID.

\section{Sistemas de información hospitalarios en el exterior}

El Leapfrog Group, creado en los Estados Unidos luego de los informes del Institute of Medicine, busca que los hospitales logren estándares que garanticen la seguridad de los servicios médicos que se ofrecen a los pacientes. Solamente 40 hospitales han cumplido hasta ahora con los tres requisitos que ha establecido el grupo, de los cuales el primero es una historia clínica digital. El beneficio para los pacientes es innegable e irá aumentando en la medida que se introduzcan en los sistemas nuevas herramientas que ayuden al personal de salud y se complementen idealmente con el uso de los sistemas de identificación por radio frecuencia para una mejor gestión en los procesos internos de la organización.

Este proceso de sistematización disminuye el error médico en la práctica habitual. Se estima que por este concepto en Estados Unidos se producen más de un millón de lesiones y 100.000 muertes anuales, las cuales se hubiesen podido evitar al mejorar la calidad en la atención de los pacientes.

A pesar de estas alarmantes cifras, se estima que la mayoría de los errores médicos no resulta en lesiones graves o muerte, sino que tienen consecuencias que pasan inadvertidas dado el estado clínico de las paciente, pero sumamente costosas para los sistemas de salud.

La implementación de tecnologías de información en el ámbito de la salud ha mostrado beneficios en la optimización de los recursos económicos de las instituciones sanitarias. En Estados Unidos algunos casos publicados han mostrado ahorros en hospitales universitarios entre el $2 \%$ y el $10 \%$ del presupuesto institucional, que representan entre US $\$ 5$ y US $\$ 26$ millones anuales, respectivamente [17].

\section{Historia clínica electrónica $\mathrm{HCE}$}

La historia clínica $(\mathrm{HC})$ se considera como el único documento válido desde los puntos de vista clínico y legal en todos los niveles de atención de salud. Con frecuencia, en el campo de la atención primaria, la historia clínica se denomina como historia de salud.

La HC es uno de los elementos fundamentales en la medicina institucional que se ejerce en nuestros días. Dicho documento es la fuente que, además de recoger todo un informe de salud, comunica el pensamiento médico, registra observaciones, diagnósticos e intervenciones que reflejan uno o varios problemas; sin embargo, su formato tradicional enfrenta diversas dificultades, que se han hecho evidentes durante la práctica diaria como es su deterioro $o$ pérdida, debido a que la historia convencional, en su formato de papel, sólo puede existir en un lugar y en un momento determinado, en condiciones determinadas, así como la presencia de una escritura pobre, ilegible e incompleta que dificulta la interpretación del mensaje que se pretende enviar.

Otra de sus limitaciones es que sólo puede contribuir de forma pasiva a la toma de decisiones y esto dificulta el análisis con fines científicos o de planeamiento de estrategias de salud.

Por esta razón se digitaliza la historia clínica, con la característica importante de poder tener todas las HC de diferentes especialistas (oftalmólogo, médico general, dentista, nutricionista y demás) en una global y con formato electrónico [18].

\section{Ventajas de la HCE sobre la HC}

La digitalización de la historia clínica permite abstraer las características fundamentales de una $\mathrm{HC}$ 
TABLA 9. NECESIDAD DEL USO DE RFID EN HOSPITALES

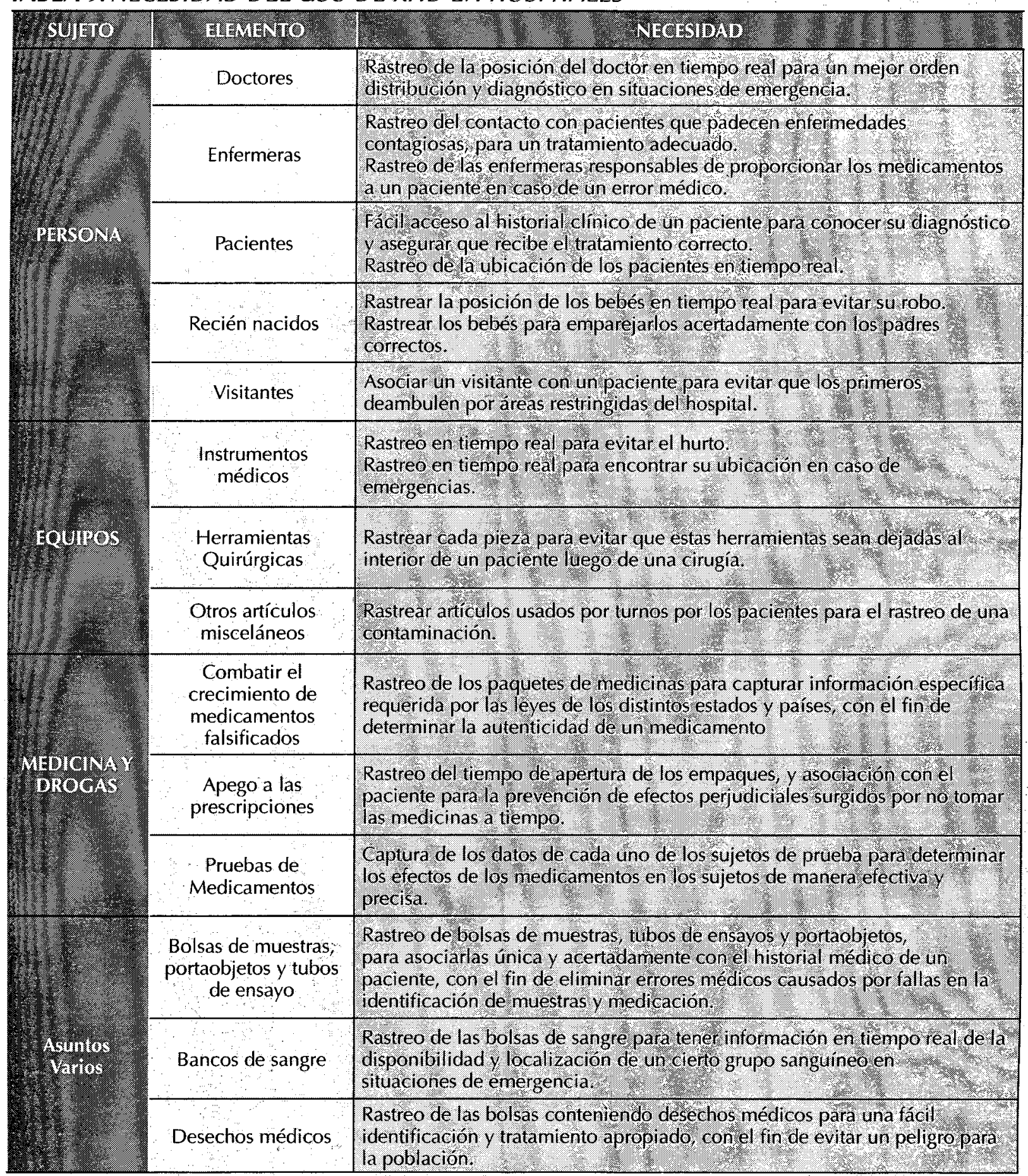

Fuente [16]

convencional con la diferencia de colocarla en un medio tecnológico que le permita una mejor gestión y control de procesos, aumentando su robustez, gra- do de privacidad y prestando una mejor eficiencia para este tipo de sistemas. 
Con el uso de la base de datos reTABLA 10. HC VS HCE

lacionales utilizadas se genera un mejor servicio para la realización de reportes y facturas, además de la posibilidad de mejorar continuamente el sistema de información por medio de la actualización periódica de los datos.

\section{Historias clínicas con RFID}

Los médicos y enfermeras cometen muchos errores en la forma en

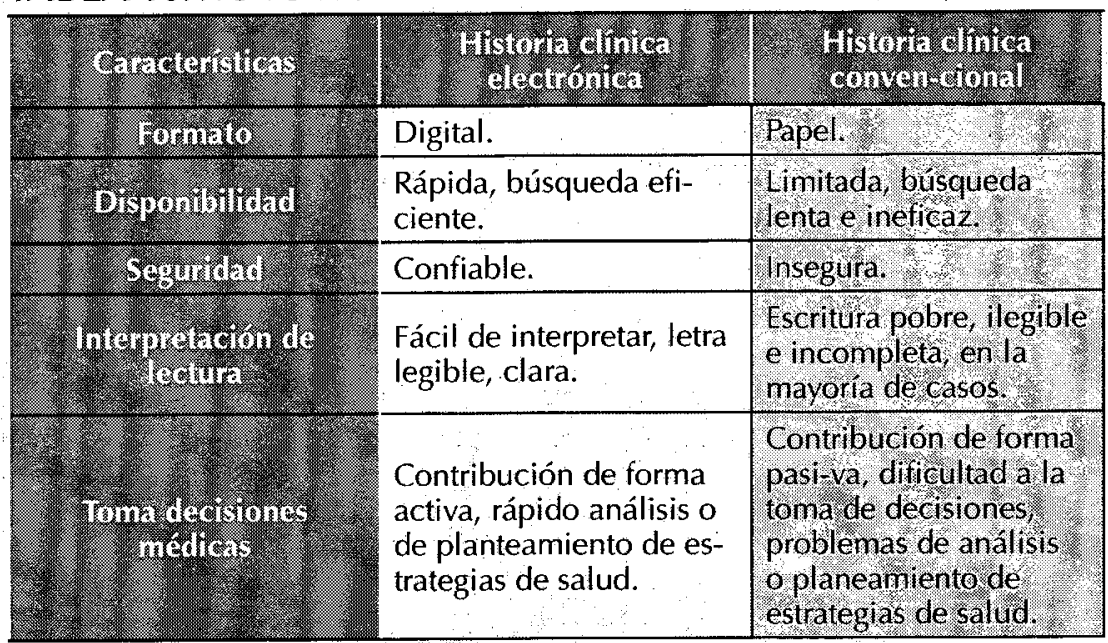
que administran los medicamentos en los pacientes, muchas veces por descuido, otras veces por información errónea, por esta razón se está desarrollando un sistema de historias clínicas con RFID de la siguiente manera:

- Se crea una historia clínica digital.

- Se le coloca un tag de manilla RFID pasiva al paciente.

- El doctor o enfermera posee una PDA que tiene un lector RFID integrado. [19]*

\section{Tag manilla RFID y lector PDA:}

El tag es colocado en la muñeca del paciente y programado con la historia clínica del mismo.

El lector PDA lo posee cada una de las enfermeras o médicos para mirar rápidamente los diagnósticos y observaciones del paciente. Esto evita errores en la cantidad y manipulación de suministros de medicina que se le suministran a los pacientes. [20]

\section{Seguridad}

La gestión integrada de los servicios sanitarios y la continuidad en los cuidados médicos requieren de mensajes y formatos, así como de una codificación

y estructura de los historiales médicos, que ofrezca interoperabilidad a los sistemas de información sanitaria con toda la seguridad que ese proceso requiere. Como parte del desarrollo, un sistema hospitalario

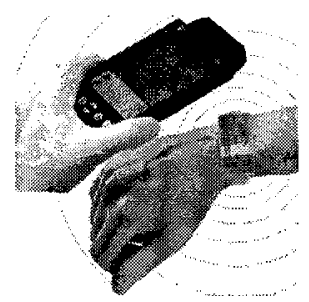

Figura 7, Tag para pacientes.

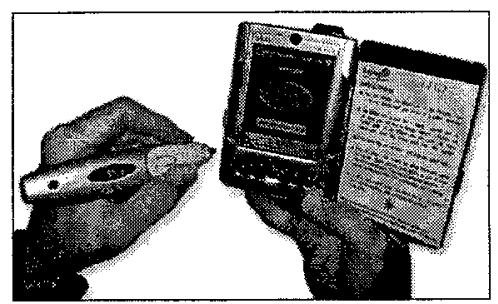

Figura 8. pda rfid tiene que contar con un conjunto de elementos de seguridad que garantice el control de acceso, confidenciabilidad, disponibilidad, integridad de datos, el no repudio y la autenticidad.

Se observa una demanda de los usuarios de sistemas abiertos, distribuidos, interconectados e interoperables, con un grado elevado de fiabilidad y requisitos de seguridad cada vez más exigentes a costos aceptables. En esa línea, los expertos indican que es necesario valorar la adopción de estándares técnicos como un elemento estratégico para la planificación, diseño, implantación, operación y mantenimiento de los sistemas de HCE. 
Ponderación de riesgos del sistema rfid

De acuerdo a un estudio desarrollado a lo largo de la investigación generamos la siguiente tabla que nos propone el nivel de riesgo que se tiene al generar una propuesta tecnológica basada en RFID para un ambiente hospitalario, en donde concluimos que la alteración de frecuencias junto con el acceso a las bases de datos del sistema son los puntos más frágiles para el funcionamiento óptimo del sistema, por lo que estamos trabajando para convertirlos en fortalezas del sistema. La idea es disminuir el riesgo de factores externos para mejorar la confianza y receptividad del proyecto ante las entidades dedicadas a la salud.

$\mathrm{V}=$ Vulnerabilidad

$\mathbf{P}=$ Probabilidad

I= Impacto

$\mathrm{R}=$ Riesgo

\section{Usos de RFID}

El alcance de la tecnología está generando oportunidades de negocios, aportes científicos y aplicaciones comerciales, sin embargo RFID no cumple solo con estas necesidades, interactúa con varios sistemas que conjuntamente crean soluciones más robustas, eficientes y sostenibles.

Las bases de datos y los sistemas de redes, son un complemento y apoyo para el sistema RFID. Para una mayor interoperatividad ya existen normatividades como Iso-15693 de lectura/escritura y Iso-14443, creando un importancia enorme en la utilización de tarjetas RFID, la utilización de estándares soportan el

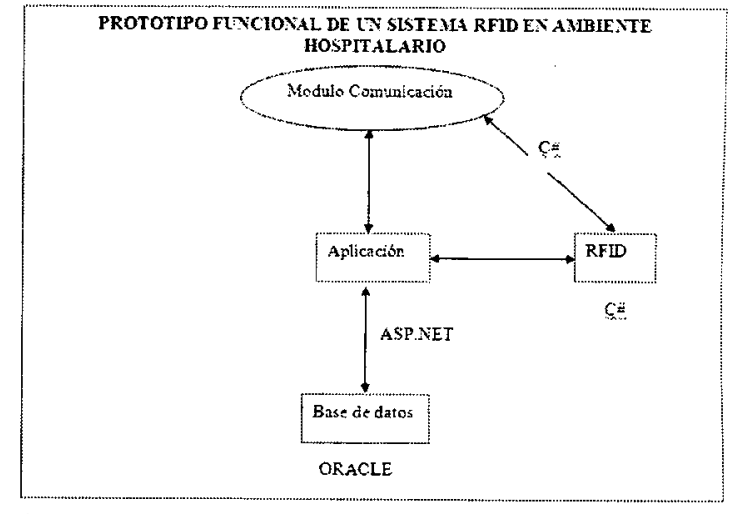

figura 9 .

trabajo de desarrolladores de aplicaciones y equipos, para crear nuevos y mejores usos de la tecnología.

La tecnología RFIDestá dando grandes pasos gracias a la incursión de aplicaciones científicas administrativas y académicas (Tabla [11])

\section{Integración}

La integración de los diferentes módulos desarrollados en las iteraciones anteriores del modelo espiral es implementada en Visual Studio.Net. El módulo de comunicación debe permitir el ingreso de los códigos de identificación captados por el lector RFID. Se utilizan tarjetas RFID, que poseen cada registro único CUID; basado en ello, la aplicación activa automáticamente el registro, de aquí depende el éxito del proceso ya que a partir de esta identificación la aplicación solicitará una contraseña de usuario para acceder a los privilegios determinados en el prototipo. 


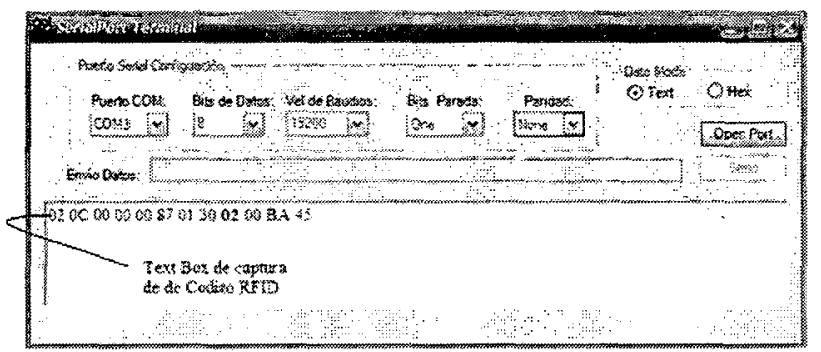

figura 10 .

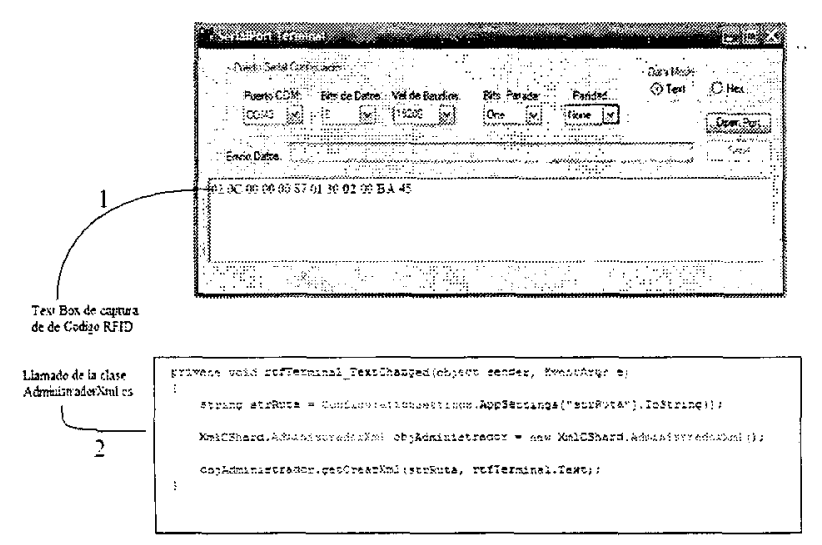

Figura 11. Integración xml.

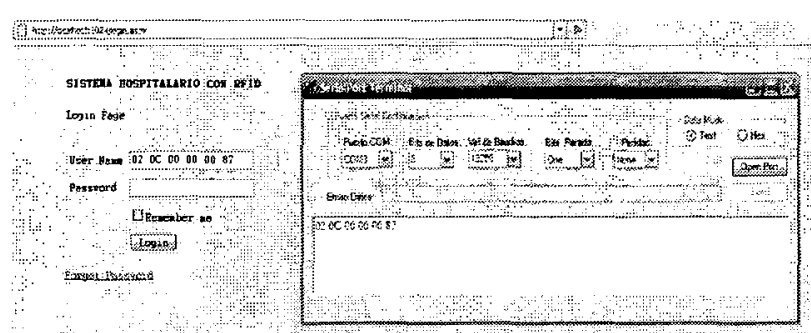

Figura 12. Integración final.

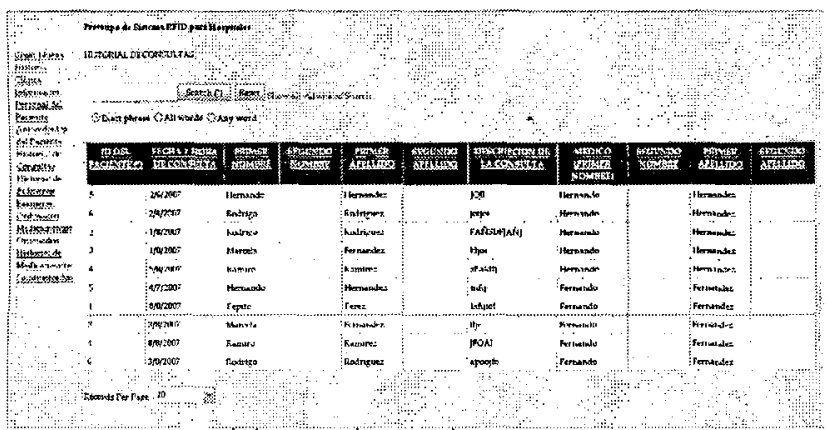

Figura 13. Historial de consultas médicas.

\section{Desarrollo}

A continuación se hace una síntesis por módulos de cada uno de los resultados obtenidos en el momento realizar la implementación de cada uno de ellos.

\section{rfid}

Se desarrolló el aplicativo de comunicación RFIDentre lector, $\operatorname{tag}$ y PC, para que de esta manera se pudiera manipular la información de envió y recepción. El lector maneja varios modos de utilización mediante la configuración de códigos hexagesimales y el protocolo RS232. Ver Figura 10.

\section{Base de datos y aplicativo ASP.NET}

Se realizó la base de datos en Oracle $10 \mathrm{~g}$, mediante el desarrollo previo de análisis y diseño guiado por el modelo en espiral.

El aplicativo se desarrollo en ASP.NET, la aplicación tiene la autenticación de pacientes y vistas para cada una de las secciones necesitadas para la historia clínica.

\section{Integración de módulos}

La integración de la $\mathrm{BD}$ (Base de datos), el aplicativo ASP.NET y el controlador de comunicación de RFID, se realizó de la siguiente manera:

1. Se creó un módulo de comunicación en XML (Extensible Markup Language), que captura el código del aplicativo de RFID y lo guarda en un archivo xml. Ver Figura 11.

2. Desde el aplicativo de ASP.NET se captura CUID guardado en el archivo XML y es mostrado en la pantalla de autenticación de usuario en el campo usuario. Desde el aplicativo de ASP.NET se captura CUID, que fue enviado por el tag y recibido por el lector en la aplicación C\#, que es almacenado en el archivo XML y es leído por el aplicativo ASP.NET. Ver figura 12.

Finalmente el código CuID, es mostrado en la página de autenticación, para poder ser consultada y administrada la historia clínica a la que esta asociada es cuidde paciente. 
Visualización de la aplicación con sus respectivas vistas de usuarios, historia clínica, consulta médica, información de pacientes entre otros. Ver Figura 13.

\section{Conclusiones}

El sistema de identificación por radiofrecuencia único con un sistema de información hospitalario genera resultados de respuesta, seguridad y almacenamiento, en los cuales la informática apenas está incursionando en nuestro país de una manera donde se garantice un aumento significativo en la prestación de servicios y se minimice el error de las entidades prestadoras de salud.

Se debe aclarar que la RFID no es un sistema de conectar è instalar, para ello hay que hacer estudios del medio, para observar los agentes que actúan en el sistema y de esa manera crear un modelo integrado para una solución efectiva del problema a tratar.

La integración, cada día mayor, a otros sistemas inalámbricos como celulares y PDA, están creando nuevas gamas de aplicaciones.en donde la RFID no ha sido implementada, la RFID está enfocada en los entornos industriales dando solución a problemas de cadena de distribución y suministro, por esta razón la parte se salud está muy poco explorada, y es ahí donde estamos haciendo gestión, en el sistema de información de RFID para historias clínicas.

El sistema de RFID en historias clínicas es un sistema que maximizará el control, atención y prestación de servicios de los pacientes. Un claro ejemplo es el momento del suministro de medicamentos en personas de alta gravedad, donde un error puede causar consecuencias traumáticas e incluso la muerte.

La digitalización de las historias clínicas es un hecho que está en proceso y que en su forma más perfecta generará una verdadera revolución en la atención de pacientes; como consecuencia, las entidades hospitalarias podrán brindar un verdadero servicio de calidad, así como lograr que los errores ocasionados por el mal manejo de la información del paciente, que pueden llegar a ocasionar grandes pérdidas, tanto económicas como de vidaś humanas, sean minimizados al máximo al poder disponer de información más confiable en cada instante.

\section{Referencias}

[1] Bhattacharya, Shaoni (2 de abril de 2005). "Electronic tags for eggs, sperm and embryos" en New Scientist.com, disponible en: http:// es.wikipedia.org/wiki/RFID

[2] Carla Lambertini, (13 de febrero de 2006) NOEL S.A. completó con éxito el piloto de tecnología RFID utilizando soluciones Oracle. La fábrica de galletas colombiana encuentra dulces recompensas en el piloto de RFID, anticipándose a las exigencias de los clientes, disponible en: http://www.oracle.com/global/lad/corporate/ press/2006_feb/noel_completo_rfid.html

[3] Pedro Alfonso Blanco (2006), Gerente de Logística de Noel S.A, Estudio de RFID en Noel, disponible en: http://www.oracle.com/global/ lad/corporate/press/206_feb/noel_completo_ rfid.html

[4]www.fundacioncorona.org.co/descargas/ PDF_publicaciones/Salud/Salud_Doc8.pdf

[5]www.fundacioncorona.org.co/descargas/ PDF_publicaciones/Salud/Salud_Doc8.pdf

[6] Casos ejemplares del uso de la tecnología como estrategia para mejorar la calidad de los servicios en el sector salud en Colombia, Revista Dinero, marzo 17 de 2006, No. 249.

[7]www.iworld.com.mx/iw_SpecialReport_ read.asp? iwid $=4413 \&$ back $=2 \&$ HistoryParam $=\mathrm{R}$ 
[8] www.gslcr.org

[9] elite.poligran.edu.co/images/5/59/Rfid.pdf

- Resultado Suplementario

[10] http://www.ericel.com/

[11]www.quiminet.com.mx/ar2/ar_\%259F

U\%258D\%251D\%253A\%25FD\%25CD\%25

DC.htm

[12] Dargan, Gaurav; Johnson,Brian; Panchalingam, Mukunthan; Stratis, Chris, The Use of Radio Frequency Identification as a Replacement for Traditional Barcoding. S.D.

[13] Tags activos vs. tags pasivos: Comparación técnica, disponible en: http://www.rfid-spain. $\mathrm{com} /$

[14] PRESSMAN, R. (2002). Ingeniería de Software: Un enfoque práctico. Quinta Edición, s.l., McGraw-Hill.

[15] ] Boehm, B. (mayo 1988). "A Spiral Model for Software Development and Enhancement", en Computer, vol. 21, n. ${ }^{\circ}$, pp. 61-72.

[16] Madhav Papp, Rohit Singhal, Ben Zoghi, (September 2004). "RFID in hospitals: Issues and Solutions." En The Consortium for the Accelerated Deployment of RFID in Distribution, s.d.

[17] Casos ejemplares del uso de la tecnología como estrategia para mejorar la calidad de los servicios en el sector salud en Colombia, Revista Dinero, marzo 17 de 2006, No. 249.

[18] Laín Entralgo P. (1998). La historia clínica. Historia y teoria del relato patográfico. Madrid: Triacastela.

[19] Historia clínica: Enciclopedia Libre Uni- versal en Español [sitio en Internet]. Disponible en:http://enciclopedia.us.es/index.php/ Historia_cl\%EDnica (Consultado: 6 de mayo del 2005).

[20] Laín Entralgo P. (1998). La historia clínica. Historia y teoria del relato patográfico. Madrid: Triacastela.

[21]www.ihg.net/java/X?cgi=lateral.rfid.Aplicaciones.pattern

\section{Gráficos}

Figura 1 http://www.ericel.com/

Figura 2 htp://www.rf-id.it/Prodotti/ProdottiPassivi.htm\#Tag

Figura 3 http://www.oracle.com/global/es/ index.html

Figura 4 http://www.discount-software.ws

Figura 5 http:// www.rfidradiotag.info/img2.jpg

Figura 6 http://www.grupo-condor.net/rfid/ que_es.htm

Figura 7 http://rfidusa.com/superstore/popup_image.php?Pid $=202$

Figura 8 http://www.rfidjournal.com/article/ articleview/1540/1/1/

Figura 9 Prototipo de un sistema RFID en el sistema hospitalario.

Figura 10 Programa RS232, aplicativo RFID.

Figura 11 Integración mediante XML, código CIUD. 
Figura 12 Integración final.

Figura 13 Historial de consultas médicas.

\section{TABLAS}

Tabla 1 Posicionamiento de RFID.

Tabla 2 Aplicación de RFID en Colombia.

Tabla 3 Diferencias entre RFID y código de barras.

Tabla 4 Ventajas y Desventajas de RFID.

Tabla 5 Componentes RFID.
Tabla 6 Características de lector RFID.

Tabla 7 Diferencias entre las clases de los Transponders.

Tabla 8 Ventajas del modelo en espiral para RFID en historias clínicas electrónicas.

Tabla 9 Necesidad del uso de RFID en hospitales. Tabla $10 \mathrm{HC}$ vs. HCE.

Tabla 11 Ponderación de riesgos RFID.

Tabla 12 Aplicación RFID. 